\title{
Fermentation and non-digestibility of Mangifera pajang fibrous pulp and its polysaccharides
}

\begin{abstract}
Populations of Bifidobacterium longum BB536, Salmonella choleraesuis JCM 6977, Escherichia coli ATCC 35922 and B. pseudocatenulatum G4 were monitored over 24 and 48 h. The digestibility of Mangifera pajang fibrous (MPF) and its polysaccharides by human gastric juice and human Ŭ-amylase were also determined. MPF and its polysaccharides increased the number of bifidobacteria but did not affect the growth of S. choleraesuis JCM 6977 or E. coli ATCC 35922; moreover, their effects were comparable to those of the commercial prebiotic inulin. M. pajang fibrous polysaccharides showed the highest nondigestibility by human gastric juice and human Ŭ-amylase compared to MPF and inulin; MPF showed a higher non-digestibility than inulin. MPF and its polysaccharides showed strong fermentation and non-digestibility properties, and thus it might be a prospective prebiotic that could be incorporated into food products.
\end{abstract}

Keyword: Prebiotic; Fermentation; Mangifera pajang; Polysaccharides 